\title{
Camera calibration from road lane markings
}

\author{
George S. K. Fung, MEMBER SPIE \\ Nelson H. C. Yung \\ Grantham K. H. Pang \\ The University of Hong Kong \\ Department of Electrical and Electronic \\ Engineering \\ Pokfulam Road, Hong Kong SAR \\ E-mail: skfung@eee.hku.hk
}

\begin{abstract}
Three-dimensional computer vision techniques have been actively studied for the purpose of visual traffic surveillance. To determine the 3-D environment, camera calibration is a crucial step to resolve the relationship between the 3-D world coordinates and their corresponding image coordinates. A novel camera calibration using the geometry properties of road lane markings is proposed. A set of equations that computes the camera parameters from the image coordinates of the road lane markings and lane width is derived. The camera parameters include pan angle, tilt angle, swing angle, focal length, and camera distance. Our results show that the proposed method outperforms the others in terms of accuracy and noise sensitivity. The proposed method accurately determines camera parameters using the appropriate camera model and it is insensitive to perturbation of noise on the calibration pattern. (๑) 2003 Society of Photo-Optical Instrumentation Engineers. [DOI: 10.1117/1.1606458]
\end{abstract}

Subject terms: camera calibration; camera model; road lane markings; visual traffic surveillance; three-dimensional computer vision; intelligent transportation systems.

Paper 020438 received Oct. 7, 2002; revised manuscript received Apr. 3, 2003; accepted for publication Apr. 14, 2003.

\section{Introduction}

Camera calibration is a crucial and preliminary step in 3-D computer vision. The key idea behind camera calibration is to develop the projection equations linking the known coordinates of a set of 3-D points and their projections, and solve for the camera parameters. ${ }^{1}$ In other words, the purpose of calibration is to establish the relationship between the 3-D world coordinates and their corresponding 2-D image coordinates as seen by the computer. ${ }^{2}$ This relationship is defined by the intrinsic and extrinsic camera parameters. The intrinsic parameters indicate the internal geometric and optical characteristics of the camera such as focal length, scale factors, and lens distortion. On the other hand, the extrinsic parameters represent the 3-D position and orientation of the camera relative to the world coordinate system. Once these camera parameters have been found, there are methods to infer 3-D information from 2-D information and vice versa. Therefore, camera calibration is required by most 3-D computer vision applications where the relationship between the 2-D image and 3-D world is needed. These applications include 3-D sensing and measurement, ${ }^{3}$ surveillance, ${ }^{4}$ manufacturing inspection, ${ }^{5}$ and automated assembly. ${ }^{6}$

Recently, we have seen growing interest in applying image processing techniques for visual traffic surveillance ${ }^{7,8}$ (VTS). Cameras used for VTS can be remotely adjusted in pan angle, tilt angle, and degree of zooming. When a camera undergoes such changes, a new set of camera parameters is required. Instead of using a predefined camera parameters, camera calibration is required to compute the changeable camera parameters ${ }^{9}$ for VTS. Therefore, it is desirable to be able to determine the set of parameters entirely from the image sequence, instead of relying on some special calibration pattern, as reported by other methods. ${ }^{10,11}$
In this paper, a novel camera calibration method for VTS is proposed. Although camera calibration methods have been developed in the last few decades, they suffer from some strict limitations. These limitations, including special camera calibration pattern, ${ }^{10}$ known camera parameters, ${ }^{12}$ and restricted camera setting ${ }^{13}$ make these methods inappropriate for VTS. In the proposed camera calibration method, we employ road lane markings, which are readily found in typical traffic scenes, as the calibration pattern. The proposed method utilizes the geometric properties of the endpoints of the road lane markings to determine the required camera parameters that govern the mapping relationship between the image and world coordinates.

The organization of this paper is as follows. Some related methods are surveyed in next section. Section 3 describes the employed perspective camera model. Section 4 describes the methodology of the proposed method. In Sec. 5 , the proposed method is evaluated by simulation results. The evaluation includes noise sensitivity and subset of camera parameters, such as omitting swing angle. Section 6 concludes the camera calibration method in terms of its advantages and limitations. The mathematical derivation of the equations is shown in the Appendix.

\section{Related Methods}

In the field of camera calibration, there are many different methods for determining the camera parameters. Some methods have been developed for special camera types, such as a single-scanline camera ${ }^{14}$ and an active or handeye camera. ${ }^{15-17}$ Moreover, some complicated methods ${ }^{18-20}$ have been developed to compensate the distortion factor of a camera. In this paper, we focus on the calibration of a single low-distortion stationary camera from a typical monocular traffic image sequence. In the 


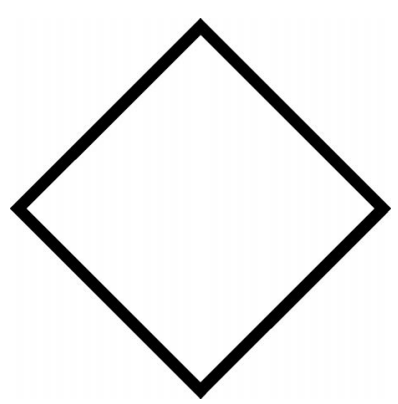

Fig. 1 Calibration pattern used by Fukui's and Courtney et al.'s methods.

following, some camera calibration methods that are closely related to our research ${ }^{10-13,21-23}$ are described.

In Ref. 13, Fukui used a diamond-shaped calibration pattern, as shown in Fig. 1, placed on the wall to determine the location of the camera with respect to the calibration pattern. The dimensions of the calibration pattern were known. The camera lens center and the calibration pattern center were set at the same height. In addition, the optical axis of the camera was required to pass through the center of the calibration pattern. The image of the calibration pattern was processed to provide two visual angles. These angles subtended the horizontal and vertical diagonals of the diamond shape. Based on these two visual angles, the unique polar coordinates of the camera relative to the calibration pattern were computed. The calibrated parameters were the distance between the camera and the center of the calibration pattern, and the angle of the camera away from the normal line of the calibration pattern. Essentially, this method was a simplified camera calibration problem, in which the camera was required to locate at the same plane as the calibration pattern and to point directly to the center of the calibration pattern. The unevenness of the floor and the distance of the camera from the calibration pattern can affect the accuracy of the method. To apply this method, the height of the camera and the calibration are required to be the same and the floor must be flat. These requirements forbid this method from general applications.

Courtney et al. ${ }^{21}$ used the same calibration pattern as Fukui but relaxed the restriction that the camera lens center must be as high as the calibration pattern center. Since the camera could be located at any height, the problem became 3-D. They split the 3-D problem into two separate 2-D problems. However, they made the assumption that the height of the camera was known.

Chou and $\mathrm{Tsai}^{22}$ used room corners as calibration pattern, as shown in Fig. 2. No doubt, their assumption was that the camera is applied indoor. A Y-shaped corner was composed of three perpendicular planes, or alternatively, of three perpendicular lines with each line being the intersection of a pair of planes. The three lines intersect at the corner point to form a Y shape. The Y shape of the corner varies with respect to the location and angle of the viewer. Under the assumption that the distance from the camera to the ceiling is known in advance, the position and orientation parameters of the camera, including the $X$ and $Y$ position parameters and the pan, tilt, and swing angles, are uniquely determined according to 3-D imaging geometry.

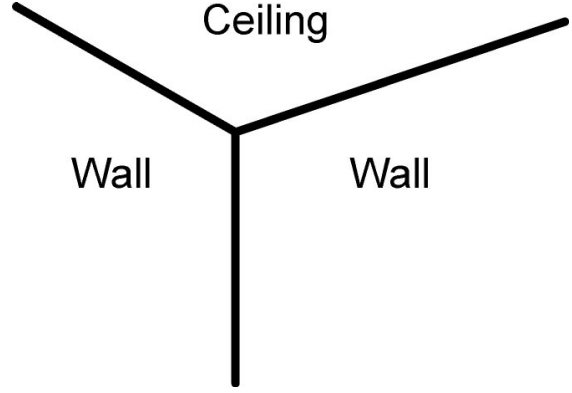

Fig. 2 Calibration pattern used by Chou and Tsai's method.

This method is effective in an indoor environment where wall-ceiling intersections are common.

In Ref. 10, a technique for 3-D camera calibration for machine vision metrology using off-the-shelf TV camera and lenses was presented. It is a two-stage technique for efficient computation of camera external position and orientation relative to object reference coordinate system as well as the effective focal length, radial lens distortion, and image scanning parameters. Tsai used the corners of 16 black-filled squares, as shown in Fig. 3, as the calibration points. These corners are located within small empty squares. Perspective projection with pin hole geometry was employed. In the first stage, the 3-D orientation and position of the camera were computed. In the second stage, an approximation of the effective focal length and height position were first computed by ignoring lens distortion. Then, an optimization scheme, such as steepest descent, was employed to solve the nonlinear equation with effective focal length, distortion coefficient, and height position being the unknowns. The major disadvantage of this approach is the inconvenience of measuring a large amount of data with respect to the world frame. It is more convenient to utilize special calibration targets to replace the measurement of individual corresponding points.

Assuming outdoor applications, Wang and Tsai ${ }^{11} \mathrm{em}-$ ployed a hexagon with three pairs of parallel edges, as shown in Fig. 4(a), as the calibration pattern. A vanishing point is formed by intersecting a pair of projected parallel edges on the ground. For a hexagon, three vanishing points are produced by the projected parallel edges on the ground. In the ideal case, a vanishing line, as depicted in Fig. 4(b), is generated by joining the vanishing points as they must be on the vanishing line. However, in practice, due to camera distortion or image processing errors, these points may not

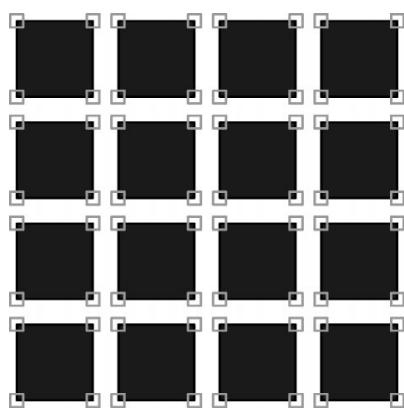

Fig. 3 Calibration pattern used by Tsai's method. 


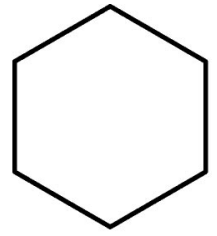

(a) Calibration pattern

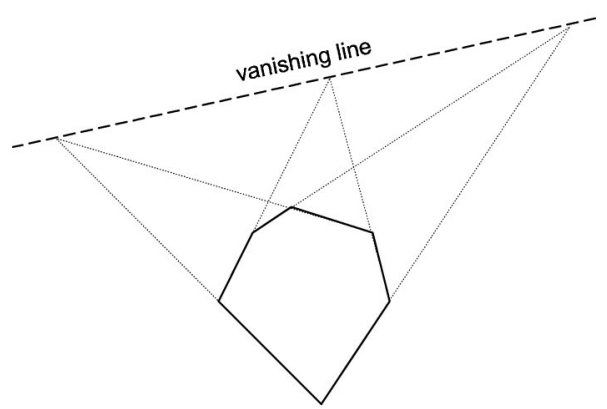

(b) Vanishing line

Fig. 4 Wang and Tsai's method.

be collinear. The line equation of the vanishing line is computed by fitting these vanishing points by least-squares error approximation. Based on the geometric hints provided by the vanishing line, the orientation, position, and the focal length of the camera could be computed. The calibrated parameters included swing angle, pan angle, tilt angle, $X-Y-Z$ coordinates, and focal length of the camera. However, it is nearly impossible to find a regular hexagon in a typical traffic scene. This means a special calibration target, a regular hexagon in this case, is required to be put on the road each time after the camera has been adjusted, that is, one or more of the camera parameters have been changed.

Bas and Crisman ${ }^{12}$ proposed an easy-to-install camera calibration method for traffic monitoring. In their method, neither measuring corresponding points nor special calibration targets were required. However, they assumed the height and the tilt angle of the camera were given beforehand. By selecting the road edges, as shown in Fig. 5, in a traffic image, the vanishing point of the road edge was used to compute the focal length and pan angle of the camera. Totally, four points, $a, b, c$, and $d$ as depicted in Fig. 5, were required in their approach. Obviously, using predefined parameters of the camera is not practical in applications where one or more of the camera parameters can be changed.

$\mathrm{Lai}^{23}$ used road lanes or edges for camera calibration. Since the height and the tilt angle of the camera could be determined on the fly, his approach was more flexible than the approach proposed by Bas and Crisman. As shown in Fig. 6, his method requires two parallel lines $p q$ and $r s$, similar to Bas and Crisman's method, and one additional perpendicular line, $m n$. The calibrated parameters obtained include focal length, camera height, and camera ground distance. Swing angle is ignored in this method.

Although many camera calibration methods have been reported, they provide only partial solutions to calibrate a camera for traffic surveillance. Basically, there are two ma-

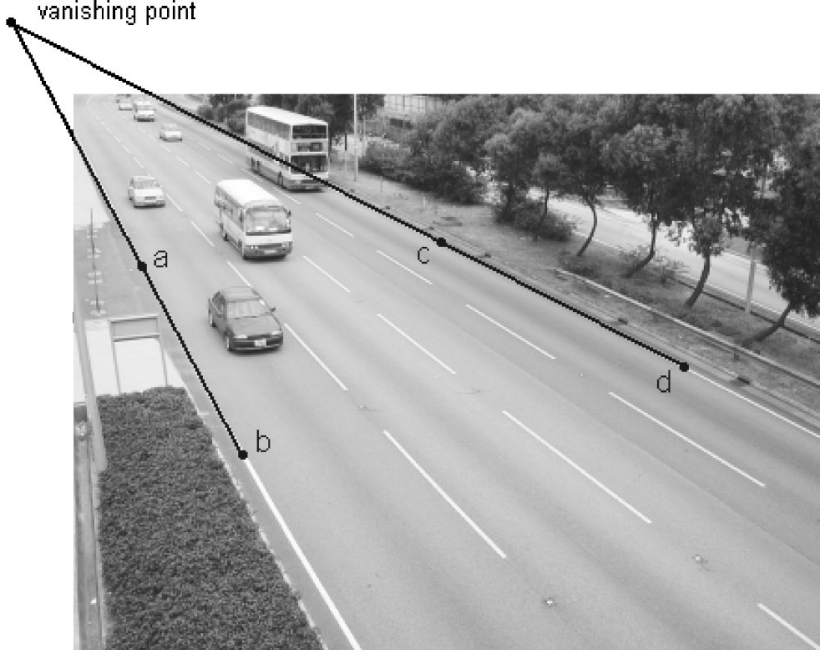

Fig. 5 Selected points used by Bas and Crisman's method.

jor limitations of these existing methods. First, in some methods, a special calibration pattern, such as diamond shape in Ref. 13 or a hexagon shape in Ref. 11, is required. These shapes can hardly be found in typical traffic scene. These special calibration patterns are required to be placed on the road surface if camera calibration is required. This is particularly impractical for closed circuit TV (CCTV) camera. Second, only a subset of the required camera parameters can be determined in some camera calibration methods for traffic surveillance cameras. In these methods, some camera parameters, such as camera height and tilt angle, have been assumed to be given beforehand. Hence, this limits the use of these methods only to cameras that can supply these parameters beforehand. Moreover, a simplified camera model is employed, in which some important camera parameters, such as swing angle, have been omitted. Omitting these camera parameters may degrade the performance of the camera calibration method.

It is desirable to develop a camera calibration method for VTS with two properties: the calibration pattern should be readily found in typical traffic scene and the camera

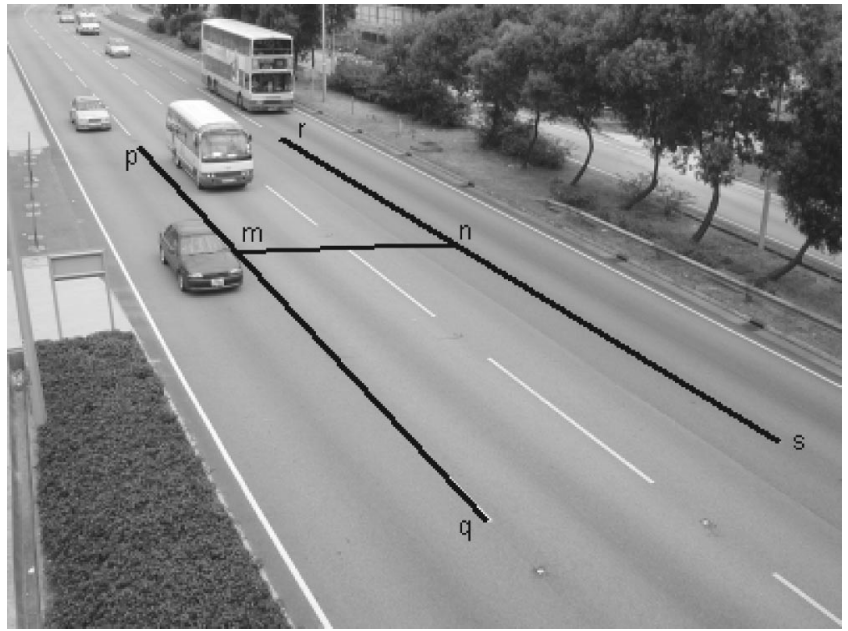

Fig. 6 Selected points used by Lai's method. 


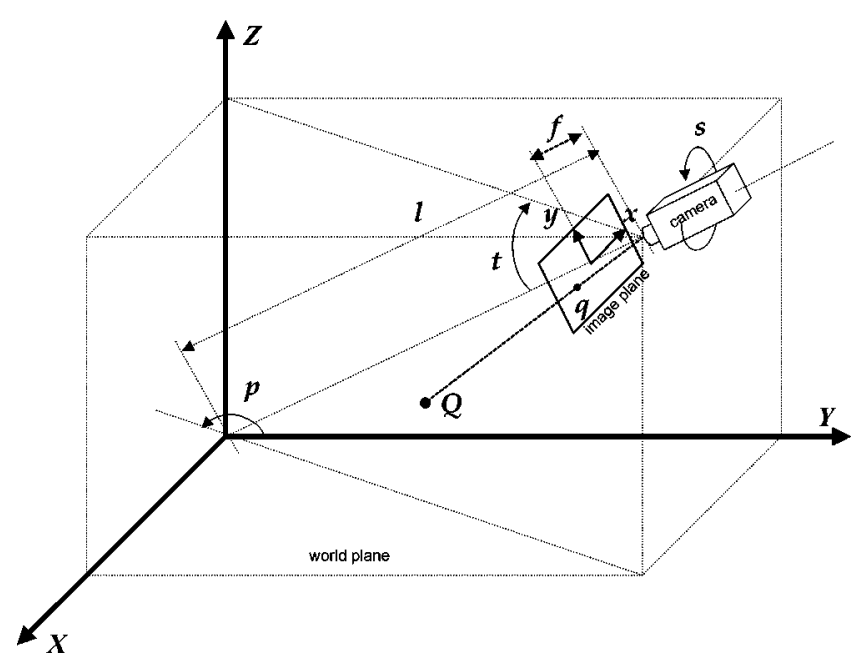

Fig. 7 Employed camera model.

model should include sufficient camera parameters to ensure accurate calibration. Road lane marking is the most common structured pattern readily found in typical traffic scene. Their geometric properties should provide sufficient information for computing the unknown camera parameters. Choosing the proper camera parameters of the camera model is crucial. In practice, ignoring camera parameters or assuming their value may result in unacceptable errors when computing other camera parameters. Clearly, adding extra camera parameters causes a dramatic increase in the complexity of equation derivation.

\section{Camera Model}

The employed camera model is depicted in Fig. 7. This model is generic enough to suit different camera settings and orientations. As shown, the relationship between the image coordinates and the world coordinates is defined in terms of pan angle $p$, tilt angle $t$, swing angle $s$, focal length $f$, and camera distance $l$. Pan angle $p$ is the horizontal angle of the optical axis of the camera with respect to the $Y$ axis of the world coordinate system. Tilt angle $t$ is the vertical angle of the optical axis of the camera with respect to the $X-Y$ plane of the world coordinate system. Swing angle $s$ is the rotation angle of the camera along its optical axis. Focal length $f$ is the distance of the image plane from the center of the camera lens along the optical axis of the camera. Camera distance $l$ is the distance of the world plane from the center of the camera lens along the optical axis of the camera.

Let $\mathbf{Q}=\left(X_{Q}, Y_{Q}, Z_{Q}\right)$ be an arbitrary point in 3-D world coordinates. Also, let $\mathbf{q}=\left(x_{q}, y_{q}\right)$ be the corresponding 2-D image coordinates of $\mathbf{Q}$. A forward mapping function $\Phi$, which defines the transform function from a point in the world coordinates to a point in the image coordinates is given as,

$\mathbf{q}=\Phi\{\mathbf{Q}\}$

By perspective transformation ${ }^{24}$ with $x_{q}=x_{q}^{*} / t_{q}^{*}$ and $y_{q}$ $=y_{q}^{*} / t_{q}^{*}$, we have $\left[\begin{array}{lll}x_{q}^{*} & y_{q}^{*} & t_{q}^{*}\end{array}\right]=\left[\begin{array}{llll}X_{Q} & Y_{Q} & Z_{Q} & 1\end{array}\right] \cdot \mathcal{T} \cdot \mathcal{R} \cdot \mathcal{P}$,

where

$\mathcal{T}=\left[\begin{array}{cccc}1 & 0 & 0 & 0 \\ 0 & 1 & 0 & 0 \\ 0 & 0 & 1 & 0 \\ -X_{\mathrm{CAM}} & -Y_{\mathrm{CAM}} & -Z_{\mathrm{CAM}} & 1\end{array}\right]$

$\mathcal{R}=\left[\begin{array}{cccc}\cos p & -\sin p & 0 & 0 \\ \sin p & \cos p & 0 & 0 \\ 0 & 0 & 1 & 0 \\ 0 & 0 & 0 & 1\end{array}\right]\left[\begin{array}{cccc}1 & 0 & 0 & 0 \\ 0 & \cos t & -\sin t & 0 \\ 0 & \sin t & \cos t & 0 \\ 0 & 0 & 0 & 1\end{array}\right]$

$\times\left[\begin{array}{cccc}\cos s & 0 & -\sin s & 0 \\ 0 & 1 & 0 & 0 \\ \sin s & 0 & \cos s & 0 \\ 0 & 0 & 0 & 1\end{array}\right]$

$\mathcal{P}=\left[\begin{array}{ccc}1 & 0 & 0 \\ 0 & 0 & 1 / f \\ 0 & 1 & 0 \\ 0 & 0 & 0\end{array}\right]$

and $\left(X_{\mathrm{CAM}}, Y_{\mathrm{CAM}}, Z_{\mathrm{CAM}}\right)$ represents the position of the camera in the world coordinates.

By substituting $\quad X_{\mathrm{CAM}}=l \sin p \cos t, \quad Y_{\mathrm{CAM}}$ $=-l \cos p \cos t, Z_{\mathrm{CAM}}=-l \sin t$ and rearranging Eq. (1), we have

$x_{q}=\frac{f\left[\begin{array}{l}X_{Q}(\cos p \cos s+\sin p \sin t \sin s) \\ +Y_{Q}(\sin p \cos s-\cos p \sin t \sin s) \\ +Z_{Q} \cos t \sin s\end{array}\right]}{-X_{Q} \sin p \cos t+Y_{Q} \cos p \cos t+Z_{Q} \sin t+l}$,

and

$y_{q}=\frac{f\left[\begin{array}{l}X_{Q}(-\cos p \sin s+\sin p \sin t \cos s) \\ +Y_{Q}(-\sin p \sin s-\cos p \sin t \cos s) \\ +Z_{Q} \cos t \cos s\end{array}\right]}{-X_{Q} \sin p \cos t+Y_{Q} \cos p \cos t+Z_{Q} \sin t+l}$.

By solving Eqs. (2) and (3), the corresponding inverse mapping $\Phi^{-1}$ is defined as

$\mathbf{Q}=\Phi^{-1}\left\{\mathbf{q}, Z_{Q}\right\}$,

where

$X_{Q}=\frac{\left[\begin{array}{l}\sin p\left(l+Z_{Q} \sin t\right)\left(x_{q} \sin s+y_{q} \cos s\right) \\ +\cos p\left(l \sin t+Z_{Q}\right)\left(x_{q} \cos s-y_{q} \sin s\right) \\ -Z_{Q} f \cos t \sin p\end{array}\right]}{x_{q} \cos t \sin s+y_{q} \cos t \cos s+f \sin t}$,

and 


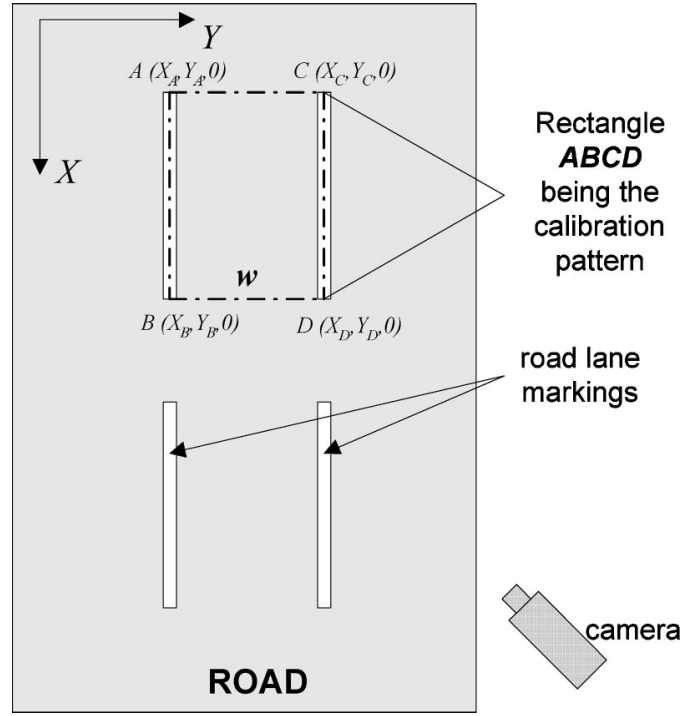

Fig. 8 Top view of the calibration pattern used.

$Y_{Q}=\frac{\left[\begin{array}{l}-\cos p\left(l+Z_{Q} \sin t\right)\left(x_{q} \sin s+y_{q} \cos s\right) \\ +\sin p\left(l \sin t+Z_{Q}\right)\left(x_{q} \cos s-y_{q} \sin s\right) \\ +Z_{Q} f \cos t \cos p\end{array}\right]}{x_{q} \cos t \sin s+y_{q} \cos t \cos s+f \sin t}$,

and $Z_{Q}$ is the assumed height of point $\mathbf{Q}$ in the world coordinates. If point $\mathbf{Q}$ lies on the $X-Y$ plane, $Z_{Q}$ becomes zero.

\section{Calibration Methodology}

Our initial assumptions are that the road under surveillance is reasonably straight and the lane markings approximate straight lines and are in parallel with each others. In practice, slight curvature is tolerable, but large road curvature that affects the straightness of the lane markings would degrade the estimated camera parameters. It is further assumed that the road surface is flat and is represented by the $X-Y$ world plane at $Z=0$, where the $X$ axis is defined along the road, and the $Y$ axis is defined perpendicular to the $X$ axis. In reality, road surfaces are slightly curved upward for drainage. This is unavoidable and will introduce a certain degree of error in our calibration. As long as the curvature of the road is not excessive, our model should still applied. Four end points of the road lane markings are arbitrarily selected to form a calibration pattern of rectangle $A B C D$, as described in Figs. 8 and 9. The world coordinates of $A$, $B, C$, and $D$ are $\left(X_{A}, Y_{A}, 0\right),\left(X_{B}, Y_{B}, 0\right),\left(X_{C}, Y_{C}, 0\right)$, $\left(X_{D}, Y_{D}, 0\right)$. The width of the lane is $w$. The typical value of $w$ is $3.6 \mathrm{~m}$, which varies for different roads. It can be automatically measured by using common vehicle with standard width, as described in Ref. 23.

With reference to Fig. 8, suppose $\overrightarrow{A B}$ and $\overrightarrow{C D}$ are parallel to the $X$ axis in the world coordinates, thus we have

Condition 1: $Y_{A}=Y_{B}$,

and

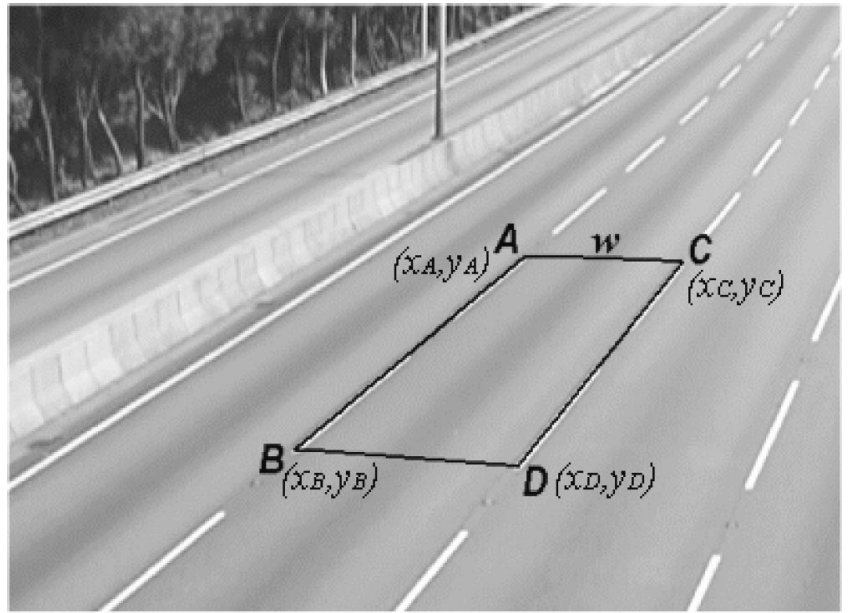

Fig. 9 Rectangle $A B C D$ view by the camera.

Condition 2: $\quad Y_{C}=Y_{D}$.

Similarly, suppose $\overrightarrow{A C}$ and $\overrightarrow{B D}$ are parallel to the $Y$ axis, we have

Condition 3: $X_{A}=X_{C}$,

and

Condition 4: $\quad X_{B}=X_{D}$.

From the lane width $w$, we have

Condition 5: $\quad Y_{C}-Y_{A}=w$.

Therefore, for a point $\mathbf{Q}$ on the road surface, where $Z_{Q}$ $=0$ in Eqs. (5) and (6), $X_{Q}$ and $Y_{Q}$ can be rewritten as

$X_{Q}=\frac{\left[\begin{array}{l}l \sin p\left(x_{q} \sin s+y_{q} \cos s\right) \\ +l \sin t \cos p\left(x_{q} \cos s-y_{q} \sin s\right)\end{array}\right]}{x_{q} \cos t \sin s+y_{q} \cos t \cos s+f \sin t}$,

and

$Y_{Q}=\frac{\left[\begin{array}{l}-l \cos p\left(x_{q} \sin s+y_{q} \cos s\right) \\ +l \sin p \sin t\left(x_{q} \cos s-y_{q} \sin s\right)\end{array}\right]}{x_{q} \cos t \sin s+y_{q} \cos t \cos s+f \sin t}$.

Using the conditions defined by Eqs. (7-11), the required camera parameters, including $p, t, s, f$, and $l$, of the camera model can be determined from the image coordinates of the four corners of the rectangle. The detailed equation derivation is illustrated in the appendix. The derived equations can be summarized as followings: 


$$
l=\frac{w\left(f \sin t+x_{A} \cos t \sin s+y_{A} \cos t \cos s\right)\left(f \sin t+x_{C} \cos t \sin s+y_{C} \cos t \cos s\right)}{\left[\begin{array}{l}
-\left(f \sin t+x_{A} \cos t \sin s+y_{A} \cos t \cos s\right) \\
\left(x_{C} \cos p \sin s-x_{C} \sin p \sin t \cos s+y_{C} \cos p \cos s+y_{C} \sin p \sin t \sin s\right) \\
+\left(f \sin t+x_{C} \cos t \sin s+y_{C} \cos t \cos s\right) \\
\left(x_{A} \cos p \sin s-x_{A} \sin p \sin t \cos s+y_{A} \cos p \cos s+y_{A} \sin p \sin t \sin s\right)
\end{array}\right]},
$$

$$
f=\frac{\chi_{B D} \cos p \cos t}{\left(\begin{array}{c}
\beta_{B D} \sin p \cos s-\beta_{B D} \cos p \sin t \sin s \\
+\alpha_{B D} \sin p \sin s+\alpha_{B D} \cos p \sin t \cos s
\end{array}\right)}
$$

$\tan p=\frac{\sin t\left[\left(\beta_{B D} \chi_{A C}-\beta_{A C} \chi_{B D}\right) \sin s+\left(\alpha_{A C} \chi_{B D}-\alpha_{B D} \chi_{A C}\right) \cos s\right]}{\left(\alpha_{B D} \chi_{A C}-\alpha_{A C} \chi_{B D}\right) \sin s+\left(\beta_{B D} \chi_{A C}-\beta_{A C} \chi_{B D}\right) \cos s}$,

$\sin t=-\left\{\frac{\left(\begin{array}{l}{\left[\left(\alpha_{B D} \chi_{A C}-\alpha_{A C} \chi_{B D}\right) \sin s+\left(\beta_{B D} \chi_{A C}-\beta_{A C} \chi_{B D}\right) \cos s\right]} \\ {\left[\left(\alpha_{C D} \chi_{A B}-\alpha_{A B} \chi_{C D}\right) \sin s+\left(\beta_{C D} \chi_{A B}-\beta_{A B} \chi_{C D}\right) \cos s\right]}\end{array}\right)}{\left(\begin{array}{l}{\left[\left(\alpha_{C D} \chi_{A B}-\alpha_{A B} \chi_{C D}\right) \cos s+\left(\beta_{A B} \chi_{C D}-\beta_{C D} \chi_{A B}\right) \sin s\right]} \\ {\left[\left(\beta_{B D} \chi_{A C}-\beta_{A C} \chi_{B D}\right) \sin s+\left(\alpha_{A C} \chi_{B D}-\alpha_{B D} \chi_{A C}\right) \cos s\right]}\end{array}\right)}\right\}^{1 / 2}$,

$$
\tan s=\frac{\left(\begin{array}{l}
-\beta_{A B} \beta_{A C} \chi_{B D} \alpha_{C D}+\beta_{A C} \alpha_{B D} \beta_{A B} \chi_{C D} \\
+\beta_{C D} \chi_{A B} \beta_{B D} \alpha_{A C}-\beta_{A B} \chi_{C D} \beta_{B D} \alpha_{A C} \\
-\beta_{C D} \beta_{B D} \chi_{A C} \alpha_{A B}-\beta_{A C} \chi_{A B} \alpha_{B D} \beta_{C D} \\
+\beta_{A B} \chi_{A C} \beta_{B D} \alpha_{C D}+\beta_{C D} \beta_{A C} \chi_{B D} \alpha_{A B}
\end{array}\right)}{\left(\begin{array}{l}
-\beta_{A B} \chi_{A C} \alpha_{B D} \alpha_{C D}+\beta_{A C} \chi_{A B} \alpha_{B D} \alpha_{C D} \\
-\beta_{A C} \alpha_{B D} \alpha_{A B} \chi_{C D}-\alpha_{A C} \chi_{B D} \beta_{C D} \alpha_{A B} \\
-\alpha_{C D} \chi_{A B} \beta_{B D} \alpha_{A C}+\beta_{A B} \alpha_{A C} \chi_{B D} \alpha_{C D} \\
+\alpha_{A B} \chi_{C D} \beta_{B D} \alpha_{A C}+\alpha_{B D} \chi_{A C} \beta_{C D} \alpha_{A B}
\end{array}\right)}
$$

where

$\alpha_{A B}=x_{B}-x_{A}, \quad \beta_{A B}=y_{B}-y_{A}, \chi_{A B}=x_{A} y_{B}-x_{B} y_{A}$,

$\alpha_{A C}=x_{C}-x_{A}, \quad \beta_{A C}=y_{C}-y_{A}, \chi_{A C}=x_{A} y_{C}-x_{C} y_{A}$,

$\alpha_{B D}=x_{D}-x_{B}, \quad \beta_{B D}=y_{D}-y_{B}, \quad \chi_{B D}=x_{B} y_{D}-x_{D} y_{B}$,

$\alpha_{C D}=x_{D}-x_{C}, \quad \beta_{C D}=y_{D}-y_{C}, \chi_{C D}=x_{C} y_{D}-x_{D} y_{C}$.

\section{Results and Discussions}

Two computer simulations were conducted to evaluate the proposed method. In the first simulation, we evaluated the proposed method in terms of the impact of swing angle against the methods developed by $\mathrm{Lai}^{23}$ and Bas. ${ }^{12}$ In the second simulation, we evaluated the sensitivity of the model to noise by comparing the proposed method against the method developed by Wang. ${ }^{11}$

\subsection{Simulation 1: Impact of Swing Angle on Accuracy}

In the methods developed by $\mathrm{Lai}^{23}$ and $\mathrm{Bas},{ }^{12}$ one of the camera parameters, swing angle, was omitted in their camera models. To evaluate the importance of swing angle in camera calibration, the error in computing other camera parameters were computed by introducing different swing angles. In the first simulation, a simulated camera with the focal length of 800 pixels was set at $25 \mathrm{~m}$ away from the world plane along the optical axis. The pan and tilt angles are set to be 110 and $-30 \mathrm{deg}$ respectively. A range of swing angles, from -5 to $5 \mathrm{deg}$, was tested for their impact on the computed focal length, pan angle, tilt angle, and camera distance.

As shown in Fig. 10, the focal length calculated by the proposed method was not affected by the change of the swing angle. It was computed as 800 pixels, as defined. However, using the methods proposed by Lai and Bas, the computed focal length would vary as the swing angle is changed. The error rates of the computed focal length are $43 \%$ for Lai's method and $5 \%$ for Bas's method when the swing angle is $-5 \mathrm{deg}$. As Bas assumed that the height and the tilt angle of the camera were already given, the computed focal length error of the method proposed was less than in Lai's method. However, this assumption highly limited the practical applicability of Bas's method as the ways to accurately estimate camera height and tilt angle were not provided.

In Fig. 11, the pan angles computed by the proposed method and Bas's method against the swing angle is depicted. Lai's method does not calculate pan angle, and therefore is not included in the comparison. Note from the result that the proposed method does not introduce error to the computed pan angle when the swing angle changes. The error of the computed pan angle is $3.2 \mathrm{deg}$ for Bas's method when swing angle is $5 \mathrm{deg}$.

In Fig. 12, the tilt angle computed by the proposed method and by Lai's method against the swing angle is depicted. Bas's method requires the tilt angle to be given, and therefore is not compared here. Note from the result that the proposed method does not introduce error to the computed tilt angle when the swing angle changes. The error of the computed tilt angle is $6.8 \mathrm{deg}$ for Lai's method when swing angle is $-5 \mathrm{deg}$.

In Fig. 13, the camera distance computed by the proposed method and Lai's method against the swing angle is

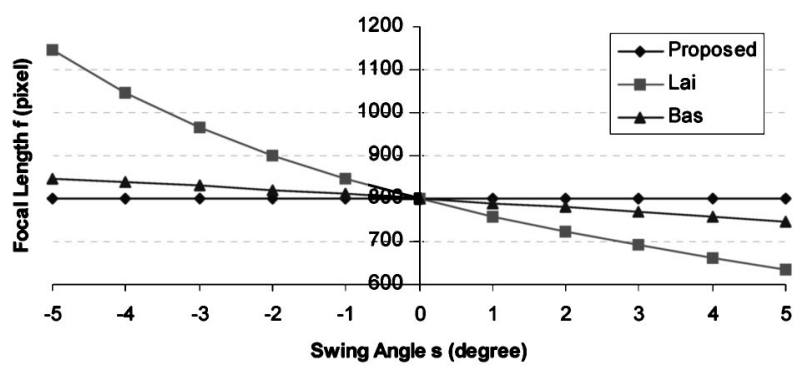

Fig. 10 Impact of swing angle $s$ on computed focal length $f$. 


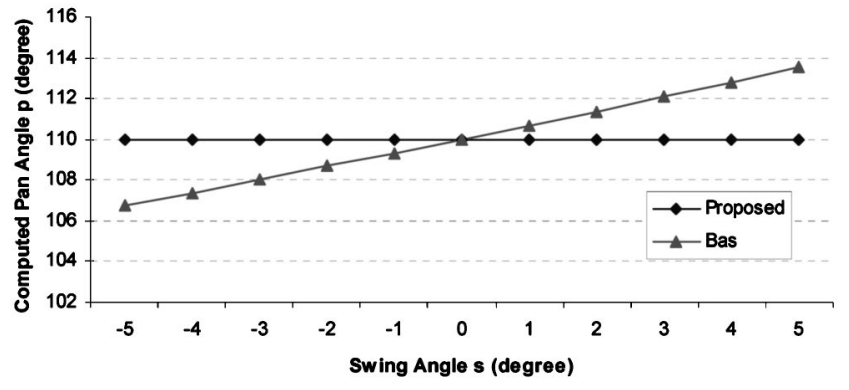

Fig. 11 Impact of swing angle $s$ on computed pan angle $p$.

depicted. Camera height is a given parameter in Bas's method and therefore is not considered. Note from the result that the proposed method does not introduce error to the computed camera distance when the swing angle changes. The error rate of the computed camera distance is $27 \%$ for Lai's method when swing angle is $-5 \mathrm{deg}$.

As shown in the results, swing angle is an important parameter in camera calibration. Omitting the swing angle in the camera model in camera calibration can cause serious errors in the computation of the camera parameters. By considering the swing angle and other parameters, the proposed method offers the most stable set of camera parameters.

\subsection{Simulation 2: Sensitivity to Noise}

In the second computer simulation, we set the simulated camera with a focal length of 800 pixels, a camera distance of $25 \mathrm{~m}$, a pan angle of $110 \mathrm{deg}$, a tilt angle of $-30 \mathrm{deg}$, and a swing angle of $-6 \mathrm{deg}$. To evaluate the sensitivity of the camera calibration method to noise, we measured the errors of the computed camera parameters, which are caused by the perturbation of the calibration pattern by different noise levels. The error of the computed camera parameter is defined as the difference between the computed and the actual values. We perturbed the calibration pattern at the image plate by adding Gaussian noise with zero mean to each pixel on the calibration pattern in both the $x$ and $y$ directions. Then, the line equation of each line of the calibration pattern was computed by a least-squares error fitting method. The noise level was adjusted by the standard deviation of the Gaussian noise, which ranges from 0.25 to 4 pixels. Since the information provided in Wang's method $^{11}$ is not sufficient to enable us to reproduce their results, we set the same camera parameters as in Ref. 11 to

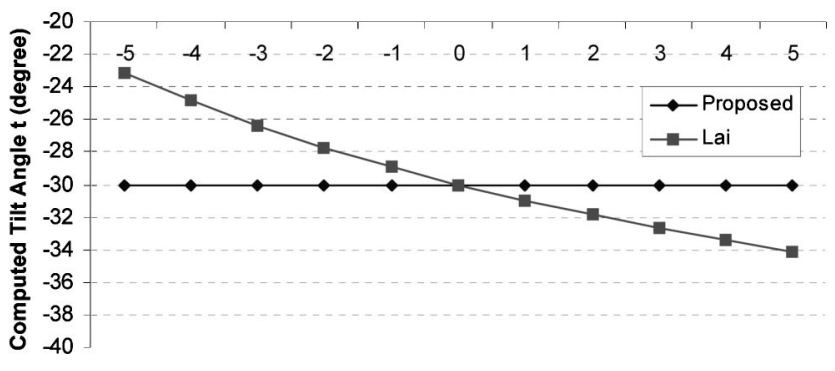

Swing Angle s (degree)

Fig. 12 Impact of swing angle $s$ on computed tilt angle $t$.

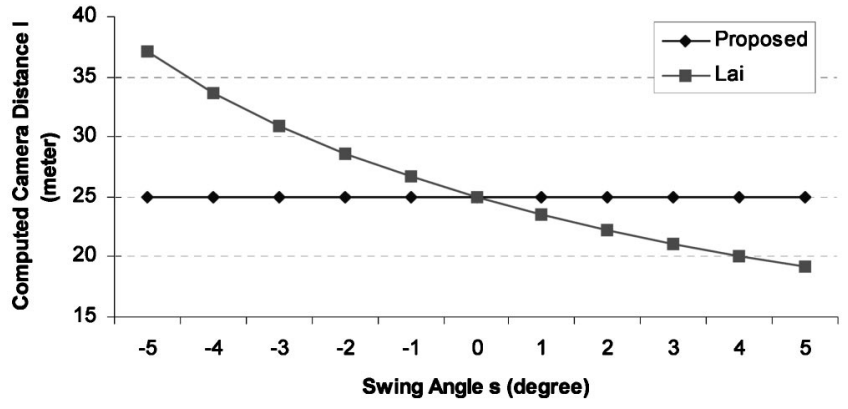

Fig. 13 Impact of swing angle $s$ on computed camera distance /.

directly compare the results. The comparisons were performed on the error of each camera parameter $p, t, s, f$, and $l$, computed by Wang's method and our proposed method at different noise levels. For each noise level, 100 simulation results were generated and averaged.

As shown in Fig. 14, we compared the error of the computed pan angle of the proposed method with that of Wang's method for different noise levels. The error of the pan angle computed by the proposed method ranges from 0.02 to $0.46 \mathrm{deg}$ for the range of noise level. However, the error of the pan angle computed by Wang's method ranges from 0.39 to $3.54 \mathrm{deg}$. In other words, Wang's method always has a higher error rate in the pan angle than the proposed method for the same noise level. For instance, the error of the pan angle computed by the proposed method is $0.46 \mathrm{deg}$ and the one computed by Wang's method is 3.54 deg, which is 6.7 times higher than the proposed method, for a noise level of 4 pixels.

As shown in Fig. 15, we compared the error of the computed tilt angle of the proposed method with that of Wang's method for different noise levels. The error of tilt angle computed by the proposed method ranges from 0.02 to 0.79 deg for the range of noise level. However, the error of the tilt angle computed by Wang's method ranges from 0.07 to $1.14 \mathrm{deg}$. Wang's method always has a higher error rate in tilt angle than the proposed method for the same noise level. For instance, the error of the tilt angle computed by the proposed method is $0.79 \mathrm{deg}$ and that computed by Wang's method is $1.14 \mathrm{deg}$, which is $44 \%$ higher than the proposed method, for a noise level of 4 pixels.

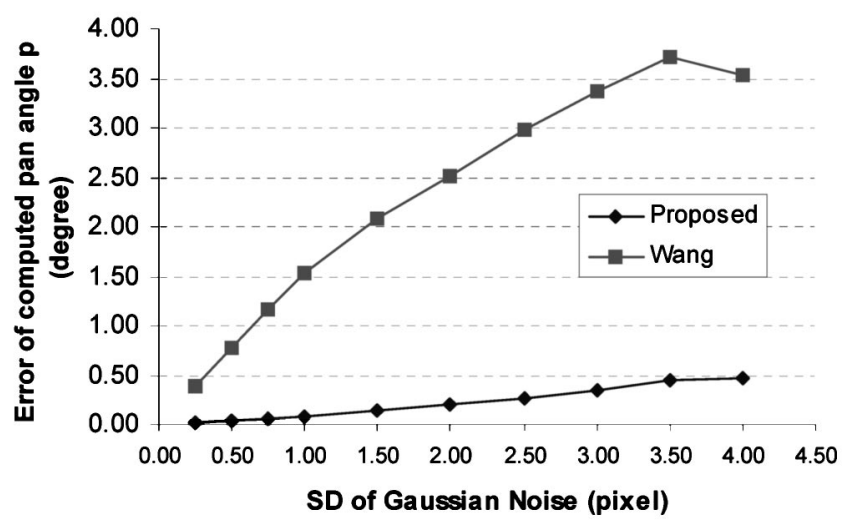

Fig. 14 Error of computed pan angle $p$ caused by Gaussian noise. 


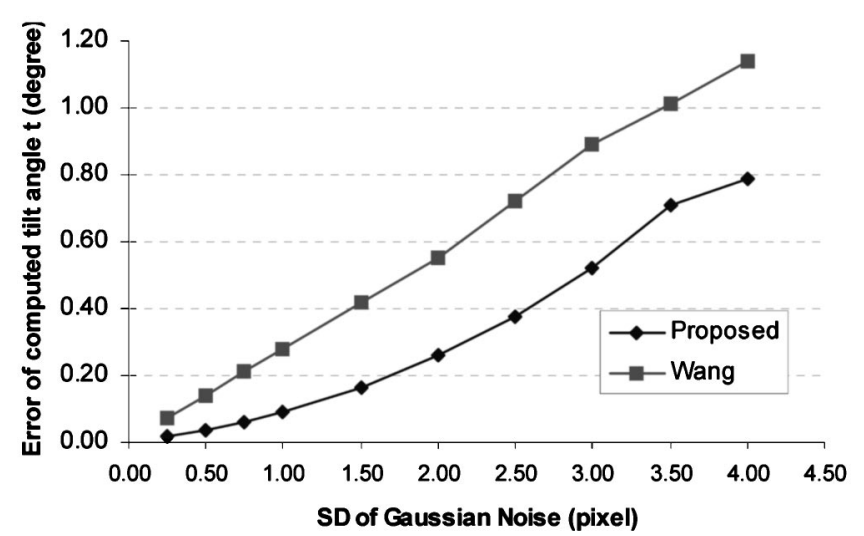

Fig. 15 Error of computed tilt angle $t$ caused by Gaussian noise.

As shown in Fig. 16, we compared the error of the computed swing angle of the proposed method with that of Wang's method for different noise levels. The error of the swing angle computed by the proposed method ranges from 0.01 to $0.30 \mathrm{deg}$ for the range of noise level. However, the error of the swing angle computed by Wang's method ranges from 0.27 to $3.21 \mathrm{deg}$. Wang's method always has a higher error rate in the swing angle than the proposed method for the same noise level. For instance, the error of the swing angle computed by the proposed method is 0.30 deg and that computed by Wang's method is $3.21 \mathrm{deg}$, which is 9.7 times higher than the proposed method for a noise level of 4 pixels.

As shown in Fig. 17, we compared the error of the computed focal length of the proposed method with that of Wang's method for different noise levels. The error of the focal length computed by the proposed method ranges from 0.80 to 55.27 pixels for the range of noise level. The error of the focal length computed by Wang's method ranges from 2.48 to 44.30 pixels. Wang's method has a higher error rate in focal length than the proposed method for noise levels below 3 pixels. For noise levels above 3 pixels, Wang's method has lower error rate in computing focal length than the proposed method. For instance, the error of the focal length computed by the proposed method is 33.48

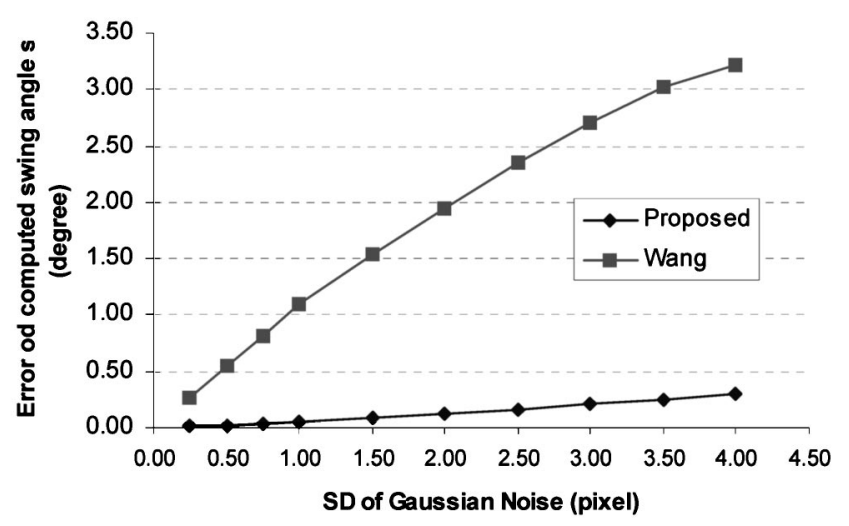

Fig. 16 Error of computed swing angle $s$ caused by Gaussian noise.

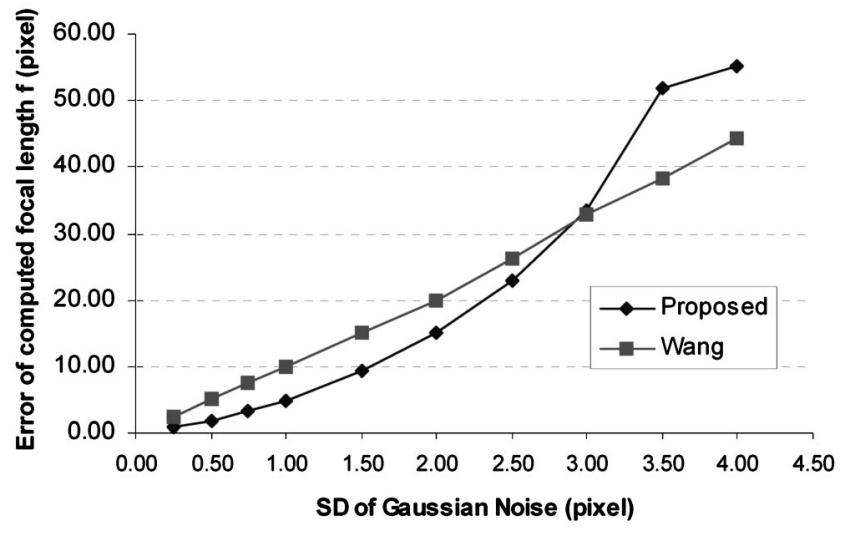

Fig. 17 Error of computed focal length $f$ caused by Gaussian noise.

pixels and that computed by Wang's method is 32.86 pixels, which is $2 \%$ lower than in the proposed method for a noise level of 3 pixels.

As shown in Fig. 18, we compared the error of computed camera distance of the proposed method with that of Wang's method for different noise levels. The error of the camera distance computed by the proposed method ranges from 0.03 to $1.90 \mathrm{~m}$ for the range of noise level. The error of the camera distance computed by Wang's method ranges from 0.98 to $11.58 \mathrm{~m}$. Wang's method always has a higher error rate in camera distance than the proposed method for the noise level range. For instance, the error of the camera distance computed by the proposed method is $1.90 \mathrm{~m}$ and that computed by Wang's method is $11.58 \mathrm{~m}$, which is 5 times higher than the proposed method for a noise level of 4 pixels.

From the results of the second simulation, we found that the proposed method is much less prone to noise than Wang's method when computing the pan angle $p$, tilt angle $t$, swing angle $s$, and camera distance $l$ under different noise levels. For focal length $f$ computation, both methods achieved comparable results.

\section{Conclusions}

VTS systems require a reliable camera calibration method. Although numerous camera calibration methods have been

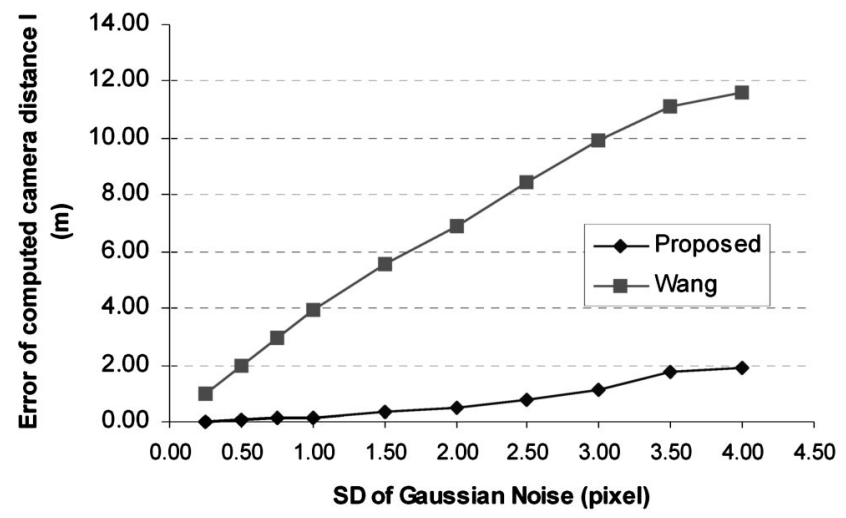

Fig. 18 Error of computed camera distance / caused by Gaussian noise. 
proposed and developed, they all suffer from some strict limitations that make them inappropriate for VTS. In this paper, a novel camera calibration method for VTS was proposed. We defined the calibration pattern from road lane markings, which are readily available in typical traffic scenes. Based on the geometric properties of the road lane markings, a set of equations that computes the camera parameters from the image coordinates of the calibration pattern and lane width was derived. The results of the computer simulations demonstrate that the proposed method outperforms the others in terms of accuracy and noise sensitivity for calculation of the camera parameters of the camera model.

In practice, a nonideal situation can reduce the accuracy of our camera calibration method. A bending road and a curved road surface are common. For a bending road, the road lane markings can be approximated by a number of short straight lines. For a curved road surface, the road surface can be divided into rectangular patches that are calibrated by individual pairs of road lane markings. Moreover, nonproperly marked, damaged or unclear road lane markings, which do not completely satisfy the assumed geometric properties, can introduce error to the camera calibration process.

\section{Appendix: Derivation}

Let the image coordinates of the four corners $A, B, C$, and $D$ of the rectangle be $\left(x_{A}, y_{A}\right),\left(x_{B}, y_{B}\right),\left(x_{C}, y_{C}\right)$, and $\left(x_{D}, y_{D}\right)$, respectively, as shown in Fig. 9. First, we substitute Eq. (13) into Eq. (11):

$$
\begin{aligned}
& \frac{\left[\begin{array}{c}
-l\left(x_{C} \cos p \sin s-x_{C} \sin p \sin t \cos s\right. \\
\left.+y_{C} \cos p \cos s+y_{C} \sin p \sin t \sin s\right)
\end{array}\right]}{f \sin t+x_{C} \cos t \sin s+y_{C} \cos t \cos s} \\
& \quad-\frac{\left[\begin{array}{c}
-l\left(x_{A} \cos p \sin s-x_{A} \sin p \sin t \cos s\right. \\
\left.+y_{A} \cos p \cos s+y_{A} \sin p \sin t \sin s\right)
\end{array}\right]}{f \sin t+x_{A} \cos t \sin s+y_{A} \cos t \cos s}=w .
\end{aligned}
$$

Then, $l$ can be obtained as follows:

$$
l=\frac{w\left(f \sin t+x_{A} \cos t \sin s+y_{A} \cos t \cos s\right)\left(f \sin t+x_{C} \cos t \sin s+y_{C} \cos t \cos s\right)}{\left[\begin{array}{l}
-\left(f \sin t+x_{A} \cos t \sin s+y_{A} \cos t \cos s\right) \\
\left(x_{C} \cos p \sin s-x_{C} \sin p \sin t \cos s+y_{C} \cos p \cos s+y_{C} \sin p \sin t \sin s\right) \\
+\left(f \sin t+x_{C} \cos t \sin s+y_{C} \cos t \cos s\right) \\
\left(x_{A} \cos p \sin s-x_{A} \sin p \sin t \cos s+y_{A} \cos p \cos s+y_{A} \sin p \sin t \sin s\right)
\end{array}\right]} .
$$

For the condition given in Eq. (7), we have

$$
\begin{aligned}
& \frac{\left[\begin{array}{c}
-l\left(x_{B} \cos p \sin s-x_{B} \sin p \sin t \cos s\right. \\
\left.+y_{B} \cos p \cos s+y_{B} \sin p \sin t \sin s\right)
\end{array}\right]}{f \sin t+x_{B} \cos t \sin s+y_{B} \cos t \cos s} \\
& \quad=\frac{\left[\begin{array}{l}
-l\left(x_{A} \cos p \sin s-x_{A} \sin p \sin t \cos s\right. \\
\left.+y_{A} \cos p \cos s+y_{A} \sin p \sin t \sin s\right)
\end{array}\right]}{f \sin t+x_{A} \cos t \sin s+y_{A} \cos t \cos s} .
\end{aligned}
$$

Then,

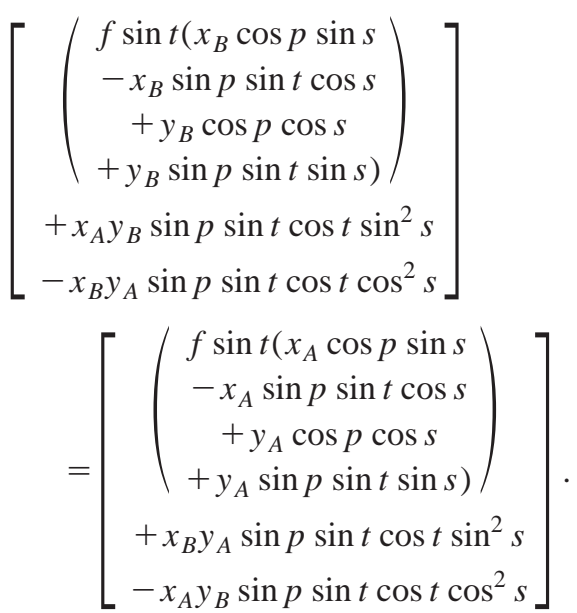

Let

$$
\begin{aligned}
& \alpha_{A B}=x_{B}-x_{A}, \quad \beta_{A B}=y_{B}-y_{A}, \quad \chi_{A B}=x_{A} y_{B}-x_{B} y_{A}, \\
& \alpha_{A C}=x_{C}-x_{A}, \quad \beta_{A C}=y_{C}-y_{A}, \quad \chi_{A C}=x_{A} y_{C}-x_{C} y_{A}, \\
& \alpha_{B D}=x_{D}-x_{B}, \quad \beta_{B D}=y_{D}-y_{B}, \quad \chi_{B D}=x_{B} y_{D}-x_{D} y_{B}, \\
& \alpha_{C D}=x_{D}-x_{C}, \quad \beta_{C D}=y_{D}-y_{C}, \quad \chi_{C D}=x_{C} y_{D}-x_{D} y_{C},
\end{aligned}
$$

and we have

$$
\begin{aligned}
& {\left[\begin{array}{c}
f\left(\alpha_{A B} \cos p \sin s-\alpha_{A B} \sin p \sin t \cos s\right. \\
\left.+\beta_{A B} \cos p \cos s+\beta_{A B} \sin p \sin t \sin s\right)
\end{array}\right]} \\
& \quad=-\chi_{A B} \sin p \cos t .
\end{aligned}
$$

Then, $f$ is given by

$$
f=\frac{-\chi_{A B} \sin p \cos t}{\left(\begin{array}{c}
\alpha_{A B} \cos p \sin s-\alpha_{A B} \sin p \sin t \cos s \\
+\beta_{A B} \cos p \cos s+\beta_{A B} \sin p \sin t \sin s
\end{array}\right)} .
$$

Similarly, from Eq. (8), we have

$$
f=\frac{-\chi_{C D} \sin p \cos t}{\left(\begin{array}{c}
\alpha_{C D} \cos p \sin s-\alpha_{C D} \sin p \sin t \cos s \\
+\beta_{C D} \cos p \cos s+\beta_{C D} \sin p \sin t \sin s
\end{array}\right)} .
$$


Using the condition given by Eq. (9), we have

$l\left(\begin{array}{c}y_{C} \sin p \cos s-y_{C} \cos p \sin t \sin s \\ +x_{C} \sin p \sin s+x_{C} \cos p \sin t \cos s\end{array}\right)$

$f \sin t+x_{C} \cos t \sin s+y_{C} \cos t \cos s$

$$
=\frac{l\left(\begin{array}{c}
y_{A} \sin p \cos s-y_{A} \cos p \sin t \sin s \\
+x_{A} \sin p \sin s+x_{A} \cos p \sin t \cos s
\end{array}\right)}{f \sin t+x_{A} \cos t \sin s+y_{A} \cos t \cos s} .
$$

Then,

$$
\begin{aligned}
& f\left(\begin{array}{c}
\beta_{A C} \sin p \cos s-\beta_{A C} \cos p \sin t \sin s \\
+\alpha_{A C} \sin p \sin s+\alpha_{A C} \cos p \sin t \cos s
\end{array}\right) \\
& =\left(\begin{array}{c}
x_{A} y_{C} \cos p \cos t \sin ^{2} s-x_{C} y_{A} \cos p \cos t \cos ^{2} s \\
-x_{C} y_{A} \cos p \cos t \sin ^{2} s+x_{A} y_{C} \cos p \cos t \cos ^{2} s
\end{array}\right) \text {. }
\end{aligned}
$$

Thus, we obtain $f$ in another form,

$$
f=\frac{\chi_{A C} \cos p \cos t}{\left(\begin{array}{c}
\beta_{A C} \sin p \cos s-\beta_{A C} \cos p \sin t \sin s \\
+\alpha_{A C} \sin p \sin s+\alpha_{A C} \cos p \sin t \cos s
\end{array}\right)} .
$$

Similarly, from Eq. (10), we have

$$
f=\frac{\chi_{B D} \cos p \cos t}{\left(\begin{array}{c}
\beta_{B D} \sin p \cos s-\beta_{B D} \cos p \sin t \sin s \\
+\alpha_{B D} \sin p \sin s+\alpha_{B D} \cos p \sin t \cos s
\end{array}\right)}
$$

The right-hand sides of Eqs. (20) and (21) are the same, and hence

$$
-\chi_{A B} \sin p \cos t
$$

$$
\begin{aligned}
& \alpha_{A B} \cos p \sin s-\alpha_{A B} \sin p \sin t \cos s \\
& \left.+\beta_{A B} \cos p \cos s+\beta_{A B} \sin p \sin t \sin s\right) \\
& =\frac{-\chi_{C D} \sin p \cos t}{\left(\begin{array}{c}
\alpha_{C D} \cos p \sin s-\alpha_{C D} \sin p \sin t \cos s \\
+\beta_{C D} \cos p \cos s+\beta_{C D} \sin p \sin t \sin s
\end{array}\right)},
\end{aligned}
$$$$
\frac{\chi_{A B}}{\left(\begin{array}{c}
\alpha_{A B} \cot p \sin s-\alpha_{A B} \sin t \cos s \\
+\beta_{A B} \cot p \cos s+\beta_{A B} \sin t \sin s
\end{array}\right)}
$$

$$
=\frac{\chi_{C D}}{\left(\begin{array}{c}
\alpha_{C D} \cot p \sin s-\alpha_{C D} \sin t \cos s \\
+\beta_{C D} \cot p \cos s+\beta_{C D} \sin t \sin s
\end{array}\right)},
$$

$$
\begin{aligned}
& \left(\begin{array}{c}
\cot p\left(\alpha_{C D} \chi_{A B} \sin s+\beta_{C D} \chi_{A B} \cos s\right) \\
+\sin t\left(-\alpha_{C D} \chi_{A B} \cos s+\beta_{C D} \chi_{A B} \sin s\right)
\end{array}\right) \\
& \quad=\left(\begin{array}{c}
\cot p\left(\alpha_{A B} \chi_{C D} \sin s+\beta_{A B} \chi_{C D} \cos s\right) \\
+\sin t\left(-\alpha_{A B} \chi_{C D} \cos s+\beta_{A B} \chi_{C D} \sin s\right.
\end{array}\right) .
\end{aligned}
$$

Then, we can obtain $p$ :

$$
\cot p=\frac{\sin t\left[\left(\alpha_{C D} \chi_{A B}-\alpha_{A B} \chi_{C D}\right) \cos s+\left(\beta_{A B} \chi_{C D}-\beta_{C D} \chi_{A B}\right) \sin s\right]}{\left(\alpha_{C D} \chi_{A B}-\alpha_{A B} \chi_{C D}\right) \sin s+\left(\beta_{C D} \chi_{A B}-\beta_{A B} \chi_{C D}\right) \cos s} .
$$

The right-hand sides of Eqs. (22) and (23) are equivalent, and hence

$\chi_{A C} \cos p \cos t$

$\overline{\left(\begin{array}{c}\beta_{A C} \sin p \cos s-\beta_{A C} \cos p \sin t \sin s \\ +\alpha_{A C} \sin p \sin s+\alpha_{A C} \cos p \sin t \cos s\end{array}\right)}$

$$
=\frac{\chi_{B D} \cos p \cos t}{\left(\begin{array}{c}
\beta_{B D} \sin p \cos s-\beta_{B D} \cos p \sin t \sin s \\
+\alpha_{B D} \sin p \sin s+\alpha_{B D} \cos p \sin t \cos s
\end{array}\right)},
$$

$\chi_{A C}\left(\begin{array}{c}\beta_{B D} \tan p \cos s-\beta_{B D} \sin t \sin s \\ +\alpha_{B D} \tan p \sin s+\alpha_{B D} \sin t \cos s\end{array}\right)$

$$
=\chi_{B D}\left(\begin{array}{c}
\beta_{A C} \tan p \cos s-\beta_{A C} \sin t \sin s \\
+\alpha_{A C} \tan p \sin s+\alpha_{A C} \sin t \cos s
\end{array}\right) .
$$

Then, we can obtain $p$ in another form:

$\tan p=\frac{\sin t\left[\left(\beta_{B D} \chi_{A C}-\beta_{A C} \chi_{B D}\right) \sin s+\left(\alpha_{A C} \chi_{B D}-\alpha_{B D} \chi_{A C}\right) \cos s\right]}{\left(\alpha_{B D} \chi_{A C}-\alpha_{A C} \chi_{B D}\right) \sin s+\left(\beta_{B D} \chi_{A C}-\beta_{A C} \chi_{B D}\right) \cos s}$.

Equating the right-hand sides of Eqs. (20) and (22), we have

$-\chi_{A B} \sin p \cos t$

$$
\begin{aligned}
& \alpha_{A B} \cos p \sin s-\alpha_{A B} \sin p \sin t \cos s \\
& \left.+\beta_{A B} \cos p \cos s+\beta_{A B} \sin p \sin t \sin s\right) \\
& =\frac{\chi_{A C} \cos p \cos t}{\left(\begin{array}{c}
\beta_{A C} \sin p \cos s-\beta_{A C} \cos p \sin t \sin s \\
+\alpha_{A C} \sin p \sin s+\alpha_{A C} \cos p \sin t \cos s
\end{array}\right)}, \\
& {\left[\begin{array}{c}
-\chi_{A B} \tan p\left(\beta_{A C} \cos s+\alpha_{A C} \sin s\right)-\chi_{A C} \cot p\left(\alpha_{A B} \sin s+\beta_{A B} \cos s\right) \\
+\left(\beta_{A C} \chi_{A B}-\beta_{A B} \chi_{A C}\right) \sin t \sin s+\left(-\alpha_{A C} \chi_{A B}+\alpha_{A B} \chi_{A C}\right) \sin t \cos s
\end{array}\right]} \\
& =0 \text {. }
\end{aligned}
$$

Equating the right-hand side of Eq. (24) and the inverse of the right-hand side of Eq. (25), we have

$$
\begin{gathered}
\frac{\sin t\left[\left(\alpha_{C D} \chi_{A B}-\alpha_{A B} \chi_{C D}\right) \cos s+\left(\beta_{A B} \chi_{C D}-\beta_{C D} \chi_{A B}\right) \sin s\right]}{\left(\alpha_{C D} \chi_{A B}-\alpha_{A B} \chi_{C D}\right) \sin s+\left(\beta_{C D} \chi_{A B}-\beta_{A B} \chi_{C D}\right) \cos s} \\
=\frac{\left(\alpha_{B D} \chi_{A C}-\alpha_{A C} \chi_{B D}\right) \sin s+\left(\beta_{B D} \chi_{A C}-\beta_{A C} \chi_{B D}\right) \cos s}{\sin t\left[\left(\beta_{B D} \chi_{A C}-\beta_{A C} \chi_{B D}\right) \sin s+\left(\alpha_{A C} \chi_{B D}-\alpha_{B D} \chi_{A C}\right) \cos s\right]}, \\
\sin ^{2} t=\frac{\left\{\begin{array}{l}
{\left[\left(\alpha_{B D} \chi_{A C}-\alpha_{A C} \chi_{B D}\right) \sin s+\left(\beta_{B D} \chi_{A C}-\beta_{A C} \chi_{B D}\right) \cos s\right]} \\
\left.\left[\left(\alpha_{C D} \chi_{A B}-\alpha_{A B} \chi_{C D}\right) \sin s+\left(\beta_{C D} \chi_{A B}-\beta_{A B} \chi_{C D}\right) \cos s\right]\right\} \\
\left\{\left(\alpha_{C D} \chi_{A B}-\alpha_{A B} \chi_{C D}\right) \cos s+\left(\beta_{A B} \chi_{C D}-\beta_{C D} \chi_{A B}\right) \sin s\right] \\
{\left[\left(\beta_{B D} \chi_{A C}-\beta_{A C} \chi_{B D}\right) \sin s+\left(\alpha_{A C} \chi_{B D}-\alpha_{B D} \chi_{A C}\right) \cos s\right]}
\end{array}\right\}}{}
\end{gathered}
$$

Since the camera is set above ground, $t$ is always negative:

$\sin t=-\left(\frac{\left\{\begin{array}{l}{\left[\left(\alpha_{B D} \chi_{A C}-\alpha_{A C} \chi_{B D}\right) \sin s+\left(\beta_{B D} \chi_{A C}-\beta_{A C} \chi_{B D}\right) \cos s\right]} \\ {\left[\left(\alpha_{C D} \chi_{A B}-\alpha_{A B} \chi_{C D}\right) \sin s+\left(\beta_{C D} \chi_{A B}-\beta_{A B} \chi_{C D}\right) \cos s\right]}\end{array}\right\}}{\left\{\begin{array}{l}{\left[\left(\alpha_{C D} \chi_{A B}-\alpha_{A B} \chi_{C D}\right) \cos s+\left(\beta_{A B} \chi_{C D}-\beta_{C D} \chi_{A B}\right) \sin s\right]} \\ {\left[\left(\beta_{B D} \chi_{A C}-\beta_{A C} \chi_{B D}\right) \sin s+\left(\alpha_{A C} \chi_{B D}-\alpha_{B D} \chi_{A C}\right) \cos s\right]}\end{array}\right\}}\right)^{1 / 2}$. 
By substituting Eqs. (24) and (25) into Eqs. (26), we have

$$
\begin{aligned}
& \left(\begin{array}{c}
\chi_{A B}\left(\beta_{A C} \cos s+\alpha_{A C} \sin s\right) \\
\left.-\left(\frac{\left[\left(\beta_{B D} \chi_{A C}-\beta_{A C} \chi_{B D}\right) \sin s+\left(\alpha_{A C} \chi_{B D}-\alpha_{B D} \chi_{A C}\right) \cos s\right]}{\left[\left(\alpha_{B D} \chi_{A C}-\alpha_{A C} \chi_{B D}\right) \sin s+\left(\beta_{B D} \chi_{A C}-\beta_{A C} \chi_{B D}\right) \cos s\right]}\right\}\right) \\
\chi_{A C}\left(\alpha_{A B} \sin s+\beta_{A B} \cos s\right) \\
\left.\left\{\frac{\left[\left(\alpha_{C D} \chi_{A B}-\alpha_{A B} \chi_{C D}\right) \cos s+\left(\beta_{A B} \chi_{C D}-\beta_{C D} \chi_{A B}\right) \sin s\right]}{\left[\left(\alpha_{C D} \chi_{A B}-\alpha_{A B} \chi_{C D}\right) \sin s+\left(\beta_{C D} \chi_{A B}-\beta_{A B} \chi_{C D}\right) \cos s\right]}\right\}\right) \\
+\left(\beta_{A C} \chi_{A B}-\beta_{A B} \chi_{A C}\right) \sin s \\
+\left(-\alpha_{A C} \chi_{A B}+\alpha_{A B} \chi_{A C}\right) \cos s
\end{array}\right)=0,
\end{aligned}
$$

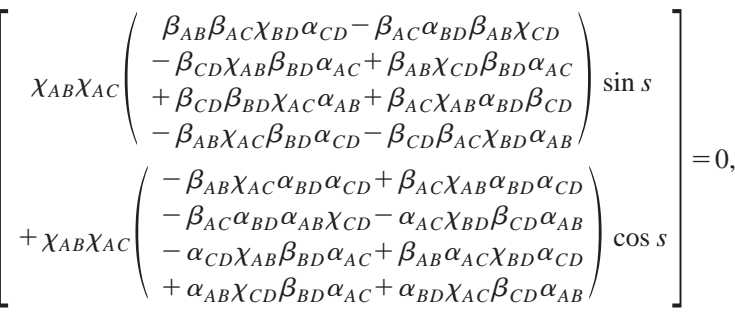

$$
\begin{aligned}
& \tan s=\frac{\left(\begin{array}{l}
-\beta_{A B} \beta_{A C} \chi_{B D} \alpha_{C D}+\beta_{A C} \alpha_{B D} \beta_{A B} \chi_{C D} \\
+\beta_{C D} \chi_{A B} \beta_{B D} \alpha_{A C}-\beta_{A B} \chi_{C D} \beta_{B D} \alpha_{A C} \\
-\beta_{C D} \beta_{B D} \chi_{A C} \alpha_{A B}-\beta_{A C} \chi_{A B} \alpha_{B D} \beta_{C D} \\
+\beta_{A B} \chi_{A C} \beta_{B D} \alpha_{C D}+\beta_{C D} \beta_{A C} \chi_{B D} \alpha_{A B}
\end{array}\right)}{\left(\begin{array}{l}
-\beta_{A B} \chi_{A C} \alpha_{B D} \alpha_{C D}+\beta_{A C} \chi_{A B} \alpha_{B D} \alpha_{C D} \\
-\beta_{A C} \alpha_{B D} \alpha_{A B} \chi_{C D}-\alpha_{A C} \chi_{B D} \beta_{C D} \alpha_{A B} \\
-\alpha_{C D} \chi_{A B} \beta_{B D} \alpha_{A C}+\beta_{A B} \alpha_{A C} \chi_{B D} \alpha_{C D} \\
+\alpha_{A B} \chi_{C D} \beta_{B D} \alpha_{A C}+\alpha_{B D} \chi_{A C} \beta_{C D} \alpha_{A B}
\end{array}\right) .}
\end{aligned}
$$

\section{References}

1. E. Trucco and A. Verri, "Camera calibration," Chap. 6 in Introductory Techniques for 3-D Computer Vision, pp. 123-138, Prentice Hall, Upper Saddle River, NJ (1998).

2. R. K. Lenz and R. Y. Tsai, "Techniques for calibration of the scale factor and image center for high accuracy 3-D machine vision metrology," IEEE Trans. Pattern Anal. Mach. Intell. 10(5), 713-720 (1988)

3. J. A. Akec, S. J. Steiner, and F. Stenger, "An experimental visual feedback control system for tracking applications using a robotic manipulator," in Proc. Annu. Conf. of the IEEE Industrial Electronics Society, Vol. 2, pp. 1125-1130 (1998).

4. E. Stringa and C. S. Regazzoni, "A novel camera calibration algorithm based on Kalman filter," in Proc. Int. Conf. on Pattern Recognition, Vol. 1, pp. 872-875 (2000).

5. J. H. M. Byne and J. A. D. W. Anderson, "CAD-based vision," in Proc. IEE Colloquium on Industrial Inspection, pp. 8/1-8/6 (1997).

6. T. S. Shen and C. H. Menq, "Automatic camera calibration for a multiple-sensor integrated coordinate measurement system," IEEE Trans. Rob. Autom. 17(4), 502-507 (2001).

7. N. Hoose, Computer Image Processing in Traffic Engineering, Research Studies Press Ltd., London (1991).

8. L. A. Klein, Sensor Technologies and Data Requirements for ITS, Artech Hose Publishers, Boston (2001).

9. N. H. C. Yung and A. H. S. Lai, "A system architecture for visual traffic surveillance," in Proc. 5th World Cong. on Intelligent Transport Systems, paper no. 3008 (1998).

10. R. Y. Tsai, "A versatile camera calibration technique for highaccuracy 3D machine vision metrology using off-the-shelf TV cameras and lenses," IEEE Trans. Rob. Autom. RA-3(4), 323-344 (1987).

11. L. L. Wang and W. H. Tsai, "Camera calibration by vanishing lines for 3-D computer vision," IEEE Trans. Pattern Anal. Mach. Intell. 13(4), 370-376 (1991)

12. E. K. Bas and J. D. Crisman, "An easy to install camera calibration for traffic monitoring," in Proc. IEEE Conf. on Intelligent Transportation Systems, pp. 362-366 (1997).

13. I. Fukui, "TV image processing to determine the position of a robot vehicle," Pattern Recogn. 14(1-6), 101-109 (1981).

14. R. Horaud, R. Mohr, and B. Lorecki, "On single-scanline camera calibration," IEEE Trans. Rob. Autom. 9(1), 71-75 (1993).

15. D. M. Song, "A self-calibration technique for active vision systems," IEEE Trans. Rob. Autom. 12(1), 114-120 (1996).

16. A. Basu and K. Raavi, "Active camera calibration using pan, tilt and roll," IEEE Trans. Syst. Man Cybernet. B 27(3), 559-566 (1997).
17. R. I. Hartley, "Self-calibration of stationary cameras," Int. J. Comput. Vis. 22(1), 5-23 (1997).

18. J. Weng, P. Cohen, and M. Herniou, "Camera calibration with distortion models and accuracy evaluation," IEEE Trans. Pattern Anal. Mach. Intell. 14(10), 965-980 (1992).

19. J. Heikkila, "Geometric camera calibration using circular control points," IEEE Trans. Pattern Anal. Mach. Intell. 22(10), 1066-1077 (2000).

20. Z. Zhang, "A flexible new technique for camera calibration," IEEE Trans. Pattern Anal. Mach. Intell. 22(11), 1330-1334 (2000).

21. J. W. Courtney, M. J. Magee, and J. K. Aggarwal, "Robot guidance using computer vision," Pattern Recogn. 17(6), 585-592 (1984).

22. W. H. Chou and W. H. Tsai, "A new approach to robot location by house corners," Pattern Recogn. 19(6), 439-451 (1986).

23. H. S. Lai, "Vehicle extraction and modeling, an effective methodology for visual traffic surveillance," PhD Thesis, Chap. 5, The University of Hong Kong (2000).

24. M. Haralick, "Using perspective transformations in scene analysis," Comput. Graph. Image Process. 13, 191-221 (1980).

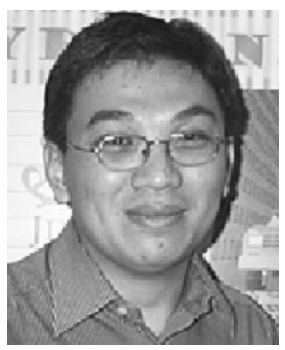

George S. K. Fung received his BSc degree from the Monash University, Australia, his MPhil degree from the Hong Kong Polytechnic University, and his $\mathrm{PhD}$ degree in electrical and electronic engineering from the University of Hong Kong (HKU) in 2003. He is affiliated to the Laboratory for Intelligent Transportation Systems $\mathrm{Re}$ search at HKU. His research interests include pattern recognition, digital image processing, intelligent transportation systems, and biomedical imaging.

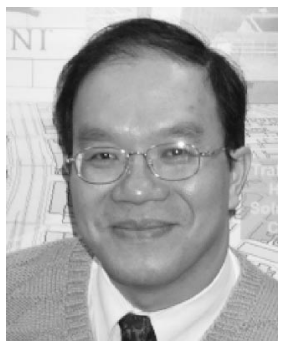

Nelson H. C. Yung received his BSc and $\mathrm{PhD}$ degrees while he was a Croucher Foundation Scholar in 1982 and 1985, respectively, from the University of Newcastle-Upon-Tyne, where he was a lecturer from 1985 until 1990. From 1990 to 1993 he was a senior research scientist with the Department of Defense, Australia. $\mathrm{He}$ joined the University of Hong Kong (HKU) in late 1993 as associate professor. $\mathrm{He}$ is the founding director of the HKU Laboratory for Intelligent Transportation Systems Research. He has coauthored a computer vision book and has published over 100 journal and conference papers in the areas of digital image processing, parallel algorithms, visual traffic surveillance, and autonomous vehicle navigation. He serves as reviewer for the IEEE Transactions of SMC, Signal Processing, the IEE Pt. G, Optical Engineering, the International Journal of Vehicle Mechanics and Mobility, Microprocessors and Microsystems, and the Robotics and Autonomous Systems Journal. He is a member of the advisory panel of the ITS Strategy Review, Transport Department, Hong Kong, and a council member of ITS-HK and Chairs the Computer Division of International Institute for Critical Infrastructures. He is a chartered electrical engineer, a member of the HKIE and IEE, and a senior member of the IEEE. He was regional secretary of the IEEE Asia-Pacific Region for 1995 to 1997. His biography has been published in Who's Who in the World since 1998.

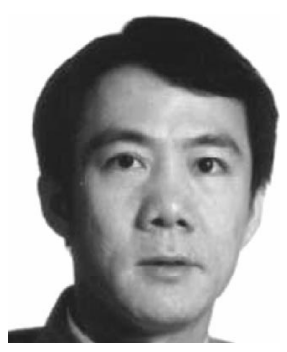

Grantham K. H. Pang received his PhD degree from the University of Cambridge in 1986. He was with the Department of Electrical and Computer Engineering, University of Waterloo, Canada, from 1986 to 1996 and joined the Department of Electrical and Electronic Engineering at the University of Hong Kong in 1996. Since 1988 he has published more than 120 technical papers and has authored or coauthored five books. His research interests include machine vision for surface defect detection, optical communications, expert systems for control system design, intelligent control, and intelligent transportation systems. 